\title{
Varieties of minimal rational tangents on Veronese double cones
}

\author{
Jun-Muk Hwang and Hosung Kim
}

\begin{abstract}
A rational curve $C$ in a nonsingular variety $X$ is standard if under the normalization $f: \mathbb{P}^{1} \rightarrow C \subset X$, the vector bundle $f^{*} T(X)$ decomposes as $\mathcal{O}(2) \oplus \mathcal{O}(1)^{p} \oplus \mathcal{O}^{q}$ for some nonnegative integers satisfying $p+q=\operatorname{dim} X-1$. For a Fano manifold $X$ of Picard number one and a general point $x \in X$, a general rational curve of minimal degree through $x$ is standard. It has been asked whether all rational curves of minimal degree through a general point $x$ are standard. Our main result is a negative answer to this question.
\end{abstract}

\section{Introduction}

Throughout this paper, we will work over the field of complex numbers. Let $X$ be a Fano manifold of Picard number one. An irreducible component $\mathcal{K}$ of the normalized space of rational curves $\operatorname{RatCurves}^{\mathrm{n}}(X)$ (see [Kol96] for a precise definition) is called a dominating family of rational curves if there is a member of $\mathcal{K}$ through a general point of $X$. We call $\mathcal{K}$ a minimal dominating family of rational curves if the degree of members of $\mathcal{K}$ with respect to $K_{X}^{-1}$ is minimal among all dominating families of rational curves. Denote by $\mathcal{K}_{x}$ the normalized space of members of $\mathcal{K}$ through a general point $x$. It is well known that $\mathcal{K}_{x}$ is a nonsingular projective variety with finitely many irreducible components. By [Keb02], the map $\tau_{x}: \mathcal{K}_{x} \rightarrow \mathbb{P} T_{x}(X)$ sending a member of $\mathcal{K}_{x}$ to its tangent direction at $x$ is a well-defined morphism finite over its image. Furthermore, by [HM04], $\tau_{x}$ is the normalization morphism of the image $\tau_{x}\left(\mathcal{K}_{x}\right)$. The image $\tau_{x}\left(\mathcal{K}_{x}\right)$ is denoted by $\mathcal{C}_{x}$ and called the variety of minimal rational tangents at $x$.

In many examples, the Fano manifold $X$ can be embedded in projective space $\mathbb{P}^{N}$ and the members of the minimal dominating family $\mathcal{K}$ are embedded as lines in $\mathbb{P}^{N}$. If this is the case, the morphism $\tau_{x}$ is an embedding and the variety of minimal rational tangents $\mathcal{C}_{x}$ is nonsingular, as explained in Proposition 1.5 of [Hwa01]. This motivates the following question.

Question 1.1 (Question 1 in [Hwa01]). For a Fano manifold $X$ of Picard number one and a minimal dominating family of rational curves, is $\tau_{x}$ an immersion, or even an embedding, at a general point $x \in X$ ?

Recall that $\tau_{x}$ is an immersion at $[C] \in \mathcal{K}_{x}$ if and only if $C$ is a standard rational curve (see

Received 17 January 2014, accepted in final form 31 July 2014.

2010 Mathematics Subject Classification 14J45

Keywords: Veronese double cone, Fano manifolds, varieties of minimal rational tangents

This journal is (C) Foundation Compositio Mathematica 2015. This article is distributed with Open Access under the terms of the Creative Commons Attribution Non-Commercial License, which permits non-commercial reuse, distribution, and reproduction in any medium, provided that the original work is properly cited. For commercial re-use, please contact the Foundation Compositio Mathematica.

Both authors are supported by National Researcher Program 2010-0020413 of NRF 


\section{VERONESE DOUBLE CONES}

[Hwa01] Proposition 1.4). So the first part of Question 1.1 is asking whether all members of $\mathcal{K}_{x}$ are standard.

It is possible to extend the setting to an arbitrary uniruled projective manifold $X$ and a locally unsplit dominating family of rational curves on $X$ (see [CD12]). The results of [HM04] and [Keb02] hold for $\tau_{x}: \mathcal{K}_{x} \rightarrow \mathbb{P} T_{x}(X)$ in this general setting and Question 1.1 can be extended as follows.

Question 1.2 (Problem 4.15 in [Hwa12]). For a uniruled projective manifold $X$ and a locally unsplit dominating family of rational curves on $X$, is $\tau_{x}$ an immersion, or even an embedding, at a general point $x \in X$ ?

Recently, Casagrande and Druel discovered a counterexample to Question 1.2 in Theorem 1.10 of [CD12]. The uniruled projective manifold $X$ in their example is a Fano manifold of Picard number three. Thus it does not give a counterexample to Question 1.1.

In the current paper, we will study the morphism $\tau_{x}$ for a class of Fano manifolds of Picard number one, Veronese double cones, and prove the following negative answer to Question 1.1.

Theorem 1.3. Let $d \geqslant 3$ be an odd positive integer, and let $f\left(x_{1}, \ldots, x_{n}, x_{n+1}\right), n \geqslant d$, be a weighted homogeneous polynomial of degree $2 d$ with respect to $\operatorname{wt}\left(x_{1}\right)=\cdots=\operatorname{wt}\left(x_{n}\right)=1$ and $\operatorname{wt}\left(x_{n+1}\right)=2$ (see Definition 2.4). Let $X^{f}$ be a Veronese double cone of dimension $n$ associated with a general choice of $f$ (see Definition 6.2). This is a Fano manifold of Picard number one (see Proposition 6.4) and there exists a minimal dominating family consisting of minimal rational curves on $X^{f}$ in the sense of Definition 6.6. If $2 d \leqslant n$, then $\tau_{x}$ at a general point $x \in X^{f}$ is not an immersion.

Our example can be viewed as an optimal counterexample to Question 1.1 in the following senses:

- When $L$ is the ample generator of $\operatorname{Pic}\left(X^{f}\right)$, minimal rational curves on $X^{f}$ have degree one with respect to $L$. In the terminology of Definition IV.2.1 of [Kol96], they form an unsplit family of rational curves. Note that in the counterexamples to Question 1.2 in [CD12], the locally unsplit dominating family is neither minimal nor unsplit (see Remark 5.4 in [CD12]).

- Suppose that a Fano manifold $X$ of Picard number one admits a morphism $\chi: X \rightarrow \mathbb{P}^{N}$ generically finite over its image such that general members of $\mathcal{K}$ are sent to lines in $\mathbb{P}^{N}$. Then by the same argument of Proposition 1.5 of [Hwa01], the morphism $\tau_{x}$ at a general point $x \in X$ is an embedding. Our $X^{f}$ admits a morphism $\chi: X \rightarrow \mathbb{P}^{N}$, finite over its image, such that general minimal rational curves on $X^{f}$ are sent to conics in $\mathbb{P}^{N}$.

To prove Theorem 1.3, we will give an explicit description (Proposition 6.7) of $\mathcal{K}_{x}$ for a general $f$ and a general point $x \in X^{f}$, as a smooth weighted complete intersection in a suitable weighted projective space. This description is of independent interest and is proved by extending some calculations from [HK13]. This enables us to express the morphism $\tau_{x}$ as an explicit projection map of this weighted complete intersection (Proposition 3.11), which cannot be an immersion if $2 d \leqslant n$ (Proposition 5.3).

\section{Notation and Convention}

1. The projectivization $\mathbb{P} V$ of a vector space $V$ is the space of one-dimensional subspaces in $V$. Given a one-dimensional subspace $U \subset V$, the symbol $[U]$ denotes the corresponding point on $\mathbb{P} V$. We will use freely the natural identification $T_{[U]}(\mathbb{P} V)=\operatorname{Hom}(U, V / U)$. 


\section{Jun-Muk Hwang AND Hosung Kim}

2. Given a vector space $V$ we will use 0 to denote sometimes the zero element and sometimes the zero subset consisting of the zero element. When we consider two or more different vector spaces at the same time, we will use the same symbol 0 to denote the zero element or the zero subset of one of the vector spaces. Usually it will be clear which vector space the zero element belongs to. For example, for the direct sum of two vector spaces $V_{1} \oplus V_{2}$, we will denote by $V_{1} \oplus 0$ the subspace given by the first factor and by $0 \oplus V_{2}$ the subspace given by the second factor.

\section{The Veronese cone}

Definition 2.1. Let $V$ be an $n$-dimensional vector space and let $R$ be a one-dimensional vector space. Consider the $\mathbb{C}^{\times}$-action on $V \oplus R$ defined by

$$
c \cdot(v, r)=\left(c v, c^{2} r\right) \quad \text { for } c \in \mathbb{C}^{\times}, v \in V \text { and } r \in R .
$$

The quotient space of $(V \oplus R) \backslash(0 \oplus 0)$ by this $\mathbb{C}^{\times}$-action is a projective variety, which we call the Veronese cone of $(V, R)$ and denote by $\mathbb{P}(V ; R)$. We denote by $\operatorname{vtx}(V ; R) \in \mathbb{P}(V ; R)$ the $\mathbb{C}^{\times}$-equivalence class of $(0 \oplus R) \backslash(0 \oplus 0)$ and call it the vertex of the Veronese cone. If $\operatorname{dim} V \geqslant 2$, the vertex is a singular point of the Veronese cone. Denote by $\mathbb{P}(V ; R)^{o}:=\mathbb{P}(V ; R) \backslash \mathbf{v t x}(V ; R)$ the complement of the vertex.

The following easy proposition explains the name 'Veronese cone'. We will skip the proof.

Proposition 2.2. (i) The map $\widehat{\iota}: V \oplus R \rightarrow \operatorname{Sym}^{2} V \oplus R$ defined by $\widehat{\iota}(v, r)=v^{2}+r$ induces a natural embedding

$$
\iota: \mathbb{P}(V ; R) \subset \mathbb{P}\left(\operatorname{Sym}^{2} V \oplus R\right) .
$$

It is an isomorphism if $\operatorname{dim} V=1$.

(ii) The image of $\iota$ is a cone over the Veronese variety $v_{2}(\mathbb{P} V) \subset \mathbb{P}\left(\mathrm{Sym}^{2} V\right)$ with vertex $\iota(\operatorname{vtx}(V ; R))=\mathbb{P}(0 \oplus R) \in \mathbb{P}\left(\operatorname{Sym}^{2} V \oplus R\right)$.

The following proposition is straightforward.

Proposition 2.3. Let $\widehat{\Psi_{V}^{R}}:(V \oplus R) \rightarrow V$ be the projection onto the first factor. It descends to a morphism $\Psi_{V}^{R}: \mathbb{P}(V ; R)^{o} \rightarrow \mathbb{P} V$, inducing a commutative diagram

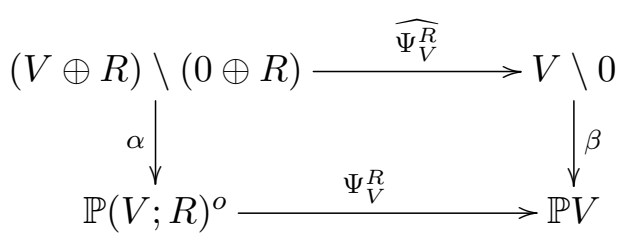

where the quotient morphisms $\alpha$ and $\beta$ induce the structures of $\mathbb{C}^{\times}$-principal bundles. Furthermore, in terms of the natural identification $T_{p}(V \oplus R)=V \oplus R$ at a point $p \in(V \oplus R) \backslash(0 \oplus R)$, we have

$$
\operatorname{Ker}\left(\mathrm{d}_{p} \widehat{\Psi_{V}^{R}}\right)=0 \oplus R \quad \text { and } \quad \operatorname{Ker}\left(\mathrm{d}_{p} \alpha\right) \cap \operatorname{Ker}\left(\mathrm{d}_{p} \widehat{\Psi_{V}^{R}}\right)=0 \oplus 0 .
$$

Definition 2.4. Let $t_{1}, \ldots, t_{N}$ be nonnegative integers. A polynomial $f\left(x_{1}, \ldots, x_{N}\right)$ in $N$ variables is said to be a weighted homogeneous polynomial of degree $k$ with respect to $\operatorname{wt}\left(x_{i}\right)=t_{i}$, $1 \leqslant i \leqslant N$, if it is of the form

$$
f\left(x_{1}, \ldots, x_{N}\right)=\sum_{t_{1} i_{1}+\cdots+t_{N} i_{N}=k} c_{i_{1}, \ldots, i_{N}} x_{1}^{i_{1}} \cdots x_{N}^{i_{N}}
$$


with coefficients $c_{i_{1}, \ldots, i_{N}} \in \mathbb{C}$.

Definition 2.5. Choose linear coordinates $x_{1}, \ldots, x_{n}$ on $V$ and a linear coordinate $x_{n+1}$ on $R$. For a weighted homogeneous polynomial $f\left(x_{1}, \ldots, x_{n}, x_{n+1}\right)$ of degree $k$ with respect to the weights $\operatorname{wt}\left(x_{1}\right)=\cdots=\operatorname{wt}\left(x_{n}\right)=1$ and $\operatorname{wt}\left(x_{n+1}\right)=2$, the zero set $\left(f\left(x_{1}, \ldots, x_{n}, x_{n+1}\right)=0\right)$ descends to a hypersurface in $\mathbb{P}(V ; R)$. Such a hypersurface is called a weighted hypersurface of degree $k$ in $\mathbb{P}(V ; R)$. This is a Weil divisor on $\mathbb{P}(V ; R)$, but not necessarily a Cartier divisor.

Proposition 2.6. There exists a connected algebraic group $G$ acting algebraically on $V \oplus R$ with the following properties:

(i) For any $g \in G$ and $(v, r) \in V \oplus R$, if $\left(v^{\prime}, r^{\prime}\right)=g \cdot(v, r)$, then $\left(c v^{\prime}, c^{2} r^{\prime}\right)=g \cdot\left(c v, c^{2} r\right)$ for any $c \in \mathbb{C}^{\times}$. In particular, the $G$-action on $V \oplus R$ descends to a $G$-action on $\mathbb{P}(V ; R)$.

(ii) The $G$-action on $\mathbb{P}(V ; R)$ is transitive on $\mathbb{P}(V ; R)^{\circ}$.

(iii) The $G$-action on $\mathbb{P}(V ; R)$ sends a weighted hypersurface of degree $k$ to a weighted hypersurface of degree $k$.

Proof. We have an action of $\mathrm{GL}(V)$ on $V \oplus R$ by $h \cdot(v, r)=(h \cdot v, r)$ for $h \in \mathrm{GL}(V)$. Also, we have an action of the vector group $\operatorname{Hom}\left(\operatorname{Sym}^{2} V, R\right)$ on $V \oplus R$, where $\varphi \in \operatorname{Hom}\left(\operatorname{Sym}^{2} V, R\right)$ acts by

$$
\varphi \cdot(v, r)=(v, \varphi(v, v)+r) .
$$

Both actions satisfy properties (i) and (iii). Then the semi-direct product $G$ of $G L(V)$ and $\operatorname{Hom}\left(\operatorname{Sym}^{2} V, R\right)$ acts on $V \oplus R$ satisfying all three conditions (i)-(iii).

Proposition 2.7. In Definition 2.5, let $S \subset \mathbb{P}(V ; R)^{\circ}$ be a nonsingular projective subvariety. For a weighted hypersurface $Z \subset \mathbb{P}(V ; R)$ of degree $k \geqslant 1$ such that $S \not \subset Z$, the intersection $S \cap Z$ is an ample divisor on $S$.

Proof. A weighted hypersurface of degree $2 d \geqslant 2$ is the pull-back of a hypersurface of degree $d$ in $\mathbb{P}\left(\mathrm{Sym}^{2} V \oplus R\right)$ by the morphism $\iota$ of Proposition 2.2. Consequently, all weighted hypersurfaces of even degree in $\mathbb{P}(V ; R)$ are ample Cartier divisors. This proves the proposition when $k$ is even.

If $k$ is odd, the Weil divisor $2 Z$ on $\mathbb{P}(V ; R)$ becomes a weighted hypersurface of degree $2 k$. Thus on $S$, the divisor $2 Z \cap S$ is an ample divisor. It follows that $Z \cap S$ is an ample divisor on $S$.

Definition 2.8. A nonsingular projective subvariety $Z \subset \mathbb{P}(V ; R)^{o}$ of codimension $m$ is called a smooth weighted complete intersection of multi-degree $\left(d_{1}, \ldots, d_{m}\right)$ if it is the scheme-theoretic intersection of $m$ weighted hypersurfaces of respective degree $d_{1}, \ldots, d_{m}$.

Proposition 2.9. Let $Z \subset \mathbb{P}(V ; R)^{\circ}$ be a smooth weighted complete intersection of multi-degree $\left(d_{1}, \ldots, d_{m}\right)$. Assume that $d_{i} \geqslant 3$ for all $i$ and $2 m \leqslant n$. Then the morphism $\left.\Psi_{V}^{R}\right|_{Z}: Z \rightarrow \mathbb{P} V$ is not an immersion.

Proof. Write $\widehat{Z}=\alpha^{-1}(Z) \subset V \oplus R$ using the notation of Proposition 2.3. By the equality

$$
\operatorname{Ker}\left(\mathrm{d}_{p} \alpha\right) \cap \operatorname{Ker}\left(\mathrm{d}_{p} \widehat{\Psi_{V}^{R}}\right)=0 \oplus 0 \quad \text { at every } p \in \widehat{Z}
$$

from Proposition 2.3, it suffices to show that $\left.\widehat{\Psi_{V}^{R}}\right|_{\widehat{Z}}: \widehat{Z} \rightarrow V$ is not an immersion. By the equality $\operatorname{Ker}\left(\mathrm{d}_{p} \widehat{\Psi_{V}^{R}}\right)=0 \oplus R$ from Proposition 2.3, it suffices to show that there exists a point $p \in \widehat{Z}$ satisfying $(0 \oplus R) \subset T_{p}(\widehat{Z})$. 


\section{Jun-Muk Hwang And Hosung Kim}

Fixing a linear coordinate system on $V \oplus R$ as in Definition 2.5, let

$$
\left\{f_{i}\left(x_{1}, \ldots, x_{n}, x_{n+1}\right), 1 \leqslant i \leqslant m\right\}
$$

be the set of weighted homogeneous polynomials of degree $d_{i}$ defining $Z$. At a point $p \in \widehat{Z}$, the tangent space $T_{p}(\widehat{Z}) \subset T_{p}(V \oplus R)=V \oplus R$ is defined by the $m$ linearly independent functionals on $V \oplus R$

$$
\sum_{\gamma=1}^{n+1} \frac{\partial f_{i}(p)}{\partial x_{\gamma}} x_{\gamma}, \quad 1 \leqslant i \leqslant m .
$$

Thus a point $p \in \widehat{Z}$ satisfies $(0 \oplus R) \subset T_{p}(\widehat{Z})$ if and only if

$$
\frac{\partial f_{i}(p)}{\partial x_{n+1}}=0 \quad \text { for all } 1 \leqslant i \leqslant m .
$$

Since $\partial f_{i} / \partial x_{n+1}$ is a weighted homogeneous polynomial of degree $d_{i}-2$, its zero set on $Z$

$$
F_{i}:=\left(\frac{\partial f_{i}}{\partial x_{n+1}}=0\right) \cap Z
$$

is either an ample divisor on $Z$ by Proposition 2.7 or $F_{i}=Z$. Since $\operatorname{dim} Z=n-m \geqslant m$, the intersection $F_{1} \cap \cdots \cap F_{m}$ is nonempty. This implies the existence of a point $p \in \widehat{Z}$ satisfying $\partial f_{i}(p) / \partial x_{n+1}=0$ for all $1 \leqslant i \leqslant m$.

\section{The Space of conics on a Veronese cone}

DeFinition 3.1. Let us use the notation of Proposition 2.2. An irreducible reduced curve $C \subset \mathbb{P}(V ; R)$ is a line (respectively, a conic) on the Veronese cone if the closure of the image $\iota\left(C \cap \mathbb{P}(V ; R)^{o}\right)$ is a line (respectively, a conic) in $\mathbb{P}\left(\mathrm{Sym}^{2} V \oplus R\right)$.

Proposition 3.2. A line on $\mathbb{P}(V ; R)$ is the closure of a fiber of the projection $\Psi_{V}^{R}$ in Proposition 2.3. More precisely, for a one-dimensional subspace $U \subset V$, the subset $\mathbb{P}(U ; R) \subset \mathbb{P}(V ; R)$ is a line in $\mathbb{P}(V ; R)$, and any line in $\mathbb{P}(V ; R)$ is of the form $\mathbb{P}(U ; R)$ for some one-dimensional subspace $U \subset V$.

Proof. It is clear that the closure of a fiber of $\Psi_{V}^{R}$ is a line and is of the form $\mathbb{P}(U ; R)$ for some one-dimensional subspace $U \subset V$. Let us check the other direction.

Consider the commuting diagram

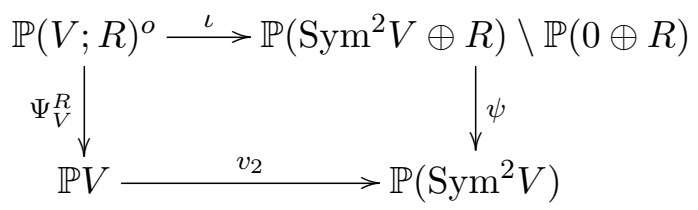

where $\psi$ denotes the projection from the vertex $\mathbb{P}(0 \oplus R)$ and $v_{2}$ denotes the second Veronese embedding. Note that the Veronese variety $v_{2}(\mathbb{P} V) \subset \mathbb{P}\left(\operatorname{Sym}^{2} V\right)$ contains no line. Thus, given a line $C \subset \mathbb{P}(V ; R)$ in Definition 3.1, the image

$$
\psi\left(\iota\left(C \cap \mathbb{P}(V ; R)^{o}\right)\right) \subset v_{2}(\mathbb{P} V)
$$

must be a point. It follows that $\Psi_{V}^{R}\left(C \cap \mathbb{P}(V ; R)^{o}\right)$ is a point and $C$ is the closure of a fiber of $\Psi_{V}^{R}$. 


\section{VERONESE DOUBLE CONES}

Notation 3.3. Let $W \subset V$ be a two-dimensional subspace and let $\varphi: \operatorname{Sym}^{2} W \rightarrow R$ be a homomorphism. Define

$$
C(W, \varphi):=\{[(w, \varphi(w, w))] \in \mathbb{P}(V ; R) \mid w \in W \backslash\{0\}\} .
$$

It is easy to check that this is a conic on $\mathbb{P}(V ; R)$ in the sense of Definition 3.1.

Proposition 3.4. A conic in $\mathbb{P}(V ; R)$ is of the form $C(W, \varphi)$ for a two-dimensional subspace $W \subset V$ and a homomorphism $\varphi: \operatorname{Sym}^{2} W \rightarrow R$. In particular, any conic is contained in $\mathbb{P}(V ; R)^{o}$.

Proof. Let $C$ be a conic on $\mathbb{P}(V ; R)$. The proper image of $C$ under the projection $\Psi_{V}^{R}$ must be a conic on the Veronese variety $v_{2}(\mathbb{P} V) \subset \mathbb{P}\left(\operatorname{Sym}^{2} V\right)$. So the image must come from a line in $\mathbb{P} V$ and be of the form $v_{2}(\mathbb{P} W) \subset \mathbb{P}\left(\operatorname{Sym}^{2} W\right)$ for some two-dimensional subspace $W \subset V$. It follows that

$$
C \subset \mathbb{P}(W ; R) \subset \mathbb{P}\left(\mathrm{Sym}^{2} W \oplus R\right) \cong \mathbb{P}^{3} .
$$

Then the linear span $P$ of $C$ in $\mathbb{P}\left(\operatorname{Sym}^{2} W \oplus R\right)$ must be a plane in $\mathbb{P}^{3}$. If $P$ contains the vertex $\operatorname{vtx}(W ; R)=\operatorname{vtx}(V ; R)$, then $P \cap \mathbb{P}(W ; R)$ must be the union of two lines (or a double line), giving a contradiction because $C \subset P \cap \mathbb{P}(W ; R)$ is assumed to be an irreducible conic. Thus $P$ does not contain the vertex. This means that its homogeneous cone $\widehat{P} \subset \mathrm{Sym}^{2} W \oplus R$ is a subspace of dimension three that does not contain $0 \oplus R$. So $\widehat{P}$ determines a homomorphism $\varphi: \operatorname{Sym}^{2} W \rightarrow R$. It is easy to check that $C=C(W, \varphi)$.

Proposition 3.5. Let $\mathcal{M} \subset \operatorname{Hilb}(\mathbb{P}(V ; R))$ be the subscheme of the Hilbert scheme parametrizing conics on $\mathbb{P}(V ; R)$. For a point $y \in \mathbb{P}(V ; R)$, let $\mathcal{M}_{y} \subset \mathcal{M}$ be the subscheme parametrizing conics through $y$. Then both $\mathcal{M}$ and $\mathcal{M}_{y}$ are smooth.

Proof. By Proposition 3.4, any conic $C$ lies in $\mathbb{P}(V ; R)^{o}$, a homogeneous variety by Proposition 2.6. Then the normal bundle $N_{C}$ of the conic in $\mathbb{P}(V ; R)^{o}$ is globally generated and consequently

$$
H^{1}\left(C, N_{C}\right)=0 \quad \text { and } \quad H^{1}\left(C, N_{C} \otimes \mathbf{m}_{y}\right)=0,
$$

where $\mathbf{m}_{y}$ is the maximal ideal of a point $y \in C$. This implies the smoothness of $\mathcal{M}$ and $\mathcal{M}_{y}$.

Proposition 3.6. Let $\operatorname{Gr}(2, V)$ be the Grassmannian of two-dimensional subspaces of $V$. Denote by $\mathcal{U} \rightarrow \operatorname{Gr}(2, V)$ the tautological bundle of rank two and by $\zeta: \operatorname{Hom}\left(\operatorname{Sym}^{2} \mathcal{U}, R\right) \rightarrow \operatorname{Gr}(2, V)$ the vector bundle whose fiber at $[W] \in \operatorname{Gr}(2, V)$ is $\operatorname{Hom}\left(\operatorname{Sym}^{2} W, R\right)$. Then we have a natural isomorphism of schemes

$$
\eta: \operatorname{Hom}\left(\operatorname{Sym}^{2} \mathcal{U}, R\right) \rightarrow \mathcal{M}
$$

sending $(W, \varphi)$ to $C(W, \varphi)$.

Proof. The association $(W, \varphi) \mapsto C(W, \varphi)$ defines a morphism $\eta$. It is easy to see that $C\left(W_{1}, \varphi_{1}\right)$ $\neq C\left(W_{2}, \varphi_{2}\right)$ if $\left(W_{1}, \varphi_{1}\right) \neq\left(W_{2}, \varphi_{2}\right)$. Thus $\eta$ is injective. Proposition 3.4 shows that $\eta$ is surjective. Since $\mathcal{M}$ is smooth by Proposition 3.5 , the bijective morphism $\eta$ must be an isomorphism of schemes.

Proposition 3.7. Let $U \subset V$ be a one-dimensional subspace. Denote by $[U] \in \mathbb{P}(V ; R)$ the point corresponding to $U$, that is, the unique $\mathbb{C}^{\times}$-equivalence class of $(U \oplus 0) \subset(V \oplus R)$. Define a submanifold $\mathcal{S}(U) \subset \operatorname{Hom}\left(\operatorname{Sym}^{2} \mathcal{U}, R\right)$ by

$$
\mathcal{S}(U):=\left\{(W, \varphi) \in \operatorname{Hom}\left(\operatorname{Sym}^{2} \mathcal{U}, R\right) \mid U \subset W, \varphi(U, U)=0\right\} .
$$

Then the restriction of $\eta$ in Proposition 3.6 gives an isomorphism $\left.\eta\right|_{\mathcal{S}(U)}: \mathcal{S}(U) \rightarrow \mathcal{M}_{[U]}$. 


\section{Jun-Muk Hwang AND Hosung Kim}

Proof. Note that a conic $C(W, \varphi)$ passes through $[U] \in \mathbb{P}(V \oplus 0) \subset \mathbb{P}(V ; R)$ if and only if $U \subset W$ and $\varphi(U, U)=0$. It follows that $\left.\eta\right|_{\mathcal{S}(U)}$ is a bijection to $\mathcal{M}_{[U]}$. Since $\mathcal{M}_{[U]}$ is a smooth subscheme of $\mathcal{M}$ by Proposition 3.5, the morphism $\left.\eta\right|_{\mathcal{S}(U)}$ is an isomorphism of schemes.

Notation 3.8. Let $U$ be a one-dimensional vector space and let $Q$ be an $(n-1)$-dimensional vector space. We have a natural inclusion of the Veronese cone

$$
\mathbb{P}\left(\operatorname{Hom}\left(\operatorname{Sym}^{2} U, R\right) ; \operatorname{Hom}\left(\operatorname{Sym}^{2} U, R\right)\right)
$$

in the Veronese cone

$$
\mathbb{P}\left(\operatorname{Hom}(U, Q) \oplus \operatorname{Hom}\left(\operatorname{Sym}^{2} U, R\right) ; \operatorname{Hom}\left(\operatorname{Sym}^{2} U, R\right)\right) .
$$

Denote by $\mathcal{V}(U, Q)$ its complement, an open subset of the latter Veronese cone. In other words, $\mathcal{V}(U, Q)$ consists of $\mathbb{C}^{\times}$-equivalence classes of elements

$$
\left.(\mu, \nu, \lambda) \in \operatorname{Hom}(U, Q) \oplus \operatorname{Hom}\left(\operatorname{Sym}^{2} U, R\right) \oplus \operatorname{Hom}\left(\operatorname{Sym}^{2} U, R\right)\right)
$$

satisfying $\mu \neq 0$ under the $\mathbb{C}^{\times}$-action defined by $c \cdot(\mu, \nu, \lambda)=\left(c \mu, c \nu, c^{2} \lambda\right)$ for $c \in \mathbb{C}^{\times}$. Denote by $\Psi_{U, Q}: \mathcal{V}(U, Q) \rightarrow \mathbb{P}\left(\operatorname{Hom}(U, Q) \oplus \operatorname{Hom}\left(\operatorname{Sym}^{2} U, R\right)\right)$ the restriction of

$$
\Psi_{\operatorname{Hom}(U, Q) \oplus \operatorname{Hom}\left(\operatorname{Sym}^{2} U, R\right)}^{\operatorname{Hom}\left(\operatorname{Sym}^{2} U, R\right)}
$$

to $\mathcal{V}(U, Q)$, sending the class of $(\mu, \nu, \lambda)$ to that of $(\mu, \nu)$.

Notation 3.9. For a point $y \in \mathbb{P}(V ; R)$, let $\tilde{\tau}_{y}: \mathcal{M}_{y} \rightarrow \mathbb{P} T_{y}(\mathbb{P}(V ; R))$ be the tangent morphism which sends a conic $C$ through $y$ to its tangent space $\mathbb{P}\left(T_{y}(C)\right) \in \mathbb{P} T_{y}(\mathbb{P}(V ; R))$.

In the next two propositions, we will give an explicit description of $\tilde{\tau}_{y}$ when $y$ is the point $[U] \in \mathbb{P}(V ; R)$ corresponding to a one-dimensional subspace $U$ of $V$. Our description depends on a choice of a complementary subspace $Q \subset V$.

Proposition 3.10. Let $V=U \oplus Q$ be the direct sum of a one-dimensional vector space $U$ and an $(n-1)$-dimensional vector space $Q$. With an element

$$
(\mu, \nu, \lambda) \in \operatorname{Hom}(U, Q) \oplus \operatorname{Hom}\left(\operatorname{Sym}^{2} U, R\right) \oplus \operatorname{Hom}\left(\operatorname{Sym}^{2} U, R\right)
$$

satisfying $\mu \neq 0$, we can associate the two-dimensional subspace $W=(U \oplus \operatorname{Im}(\mu))$ of $V$ and the element $\varphi \in \operatorname{Hom}\left(\operatorname{Sym}^{2} W, R\right)$ determined by

$$
\varphi(u, u)=0, \varphi(u, \mu(u))=\nu(u, u) \quad \text { and } \quad \varphi(\mu(u), \mu(u))=\lambda(u, u) \quad \text { for all } u \in U .
$$

This association $(\mu, \nu, \lambda) \mapsto(W, \varphi)$ induces an isomorphism $\theta: \mathcal{V}(U, Q) \rightarrow \mathcal{S}(U) \cong \mathcal{M}_{[U]}$.

Proof. It is straightforward to check that two elements $\left(\mu_{1}, \nu_{1}, \lambda_{1}\right)$ and $\left(\mu_{2}, \nu_{2}, \lambda_{2}\right)$ determine the same $(W, \varphi)$ if and only if $\mu_{1}=c \mu_{2}, \nu_{1}=c \nu_{2}, \lambda_{1}=c^{2} \lambda_{2}$. This show that the morphism $\theta: \mathcal{V}(U, Q) \rightarrow \mathcal{S}(U)$ is well defined and injective. Given $(W, \varphi) \in \mathcal{S}(U)$, let $(\mu, \nu, \lambda), \mu \neq 0$, be defined by

(i) $\mu$ is a nonzero element of $\operatorname{Hom}(U, W \cap Q) \subset \operatorname{Hom}(U, Q)$;

(ii) $\nu(u, u)=\varphi(u, \mu(u))$; and

(iii) $\lambda(u, u)=\varphi(\mu(u), \mu(u))$.

Then clearly $\theta([(\mu, \nu, \lambda)])=(W, \varphi)$. Thus $\theta$ is an isomorphism. 


\section{VERONESE DOUBLE CONES}

Proposition 3.11. In the setting of Proposition 3.10, the hypersurface $\mathbb{P}(V \oplus 0) \subset \mathbb{P}(V ; R)$ and the line $\mathbb{P}(U ; R) \subset \mathbb{P}(V ; R)$ intersect transversally at $[U]$, giving a natural isomorphism

$$
T_{[U]}(\mathbb{P}(V ; R))=T_{[U]}(\mathbb{P} V) \oplus T_{[U]}(\mathbb{P}(U ; R))=\operatorname{Hom}(U, Q) \oplus \operatorname{Hom}\left(\operatorname{Sym}^{2} U, R\right) .
$$

Here, the natural isomorphism $T_{[U]}(\mathbb{P}(U ; R))=\operatorname{Hom}\left(\operatorname{Sym}^{2} U, R\right)$ comes from the embedding $\iota: \mathbb{P}(U ; R) \cong \mathbb{P}\left(\operatorname{Sym}^{2} U \oplus R\right)$ in Proposition 2.2(i). Under the isomorphism (3.1), the tangent space of a conic $[C(W, \varphi)] \in \mathcal{M}_{[U]}$ corresponds to the one-dimensional subspace

$$
\mathcal{T}(W, \varphi) \subset \operatorname{Hom}(U, Q) \oplus \operatorname{Hom}\left(\operatorname{Sym}^{2} U, R\right)
$$

defined by

$$
\mathcal{T}(W, \varphi):=\{(\mu, \nu) \mid \mu \in \operatorname{Hom}(U, W \cap Q) \text { and } \nu(u, u)=\varphi(u, \mu(u))\} .
$$

Thus we have a commuting diagram

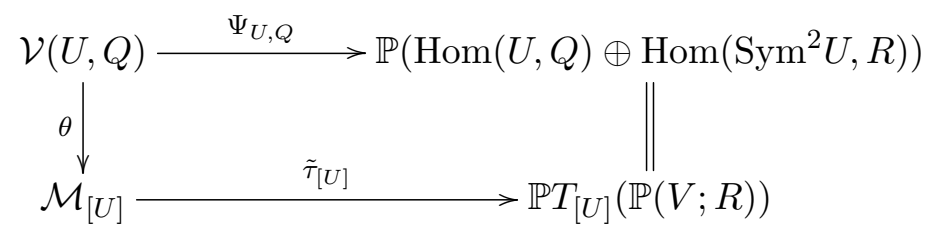

where the morphisms are as in Notation 3.9, Notation 3.8 and Proposition 3.10.

Proof. Note that if $(\mu, \nu)$ is an element of $\mathcal{T}(W, \varphi)$, then so is $(c \mu, c \nu)$ for any $c \in \mathbb{C}$. Thus $\mathcal{T}(W, \varphi)$ is indeed a one-dimensional vector space. To check that it corresponds to $T_{[U]}(C(W, \varphi))$, fix nonzero vectors $u \in U$ and $q \in Q \cap W$. The arc

$$
\{[u+t q, \varphi(u+t q, u+t q)] \in \mathbb{P}(V ; R) \mid t \in \mathbb{C}\}
$$

lies in $C(W, \varphi)$. Under the embedding $\iota$, this arc is sent to

$$
\left\{\left[(u+t q)^{2}+\varphi(u+t q, u+t q)\right] \in \mathbb{P}\left(\operatorname{Sym}^{2} V \oplus R\right) \mid t \in \mathbb{C}\right\} .
$$

Since $\varphi(u, u)=0$, this is tangent to the line in $\mathbb{P}\left(\operatorname{Sym}^{2} V \oplus R\right)$ corresponding to the twodimensional subspace

$$
\mathbb{C} u^{\otimes 2}+\mathbb{C}(u \otimes q+\varphi(u, q)) \subset \operatorname{Sym}^{2} V \oplus R .
$$

The tangent to this line at $\left[u^{\otimes 2}\right]$ is the one-dimensional subspace of

$$
T_{\left[\operatorname{Sym}^{2} U\right]}\left(\mathbb{P}\left(\operatorname{Sym}^{2} V \oplus R\right)\right)=\operatorname{Hom}\left(\operatorname{Sym}^{2} U,(U \otimes Q) \oplus \operatorname{Sym}^{2} Q \oplus R\right)
$$

spanned by the homomorphism $u^{\otimes 2} \mapsto u \otimes q+\varphi(u, q)$. Via the natural inclusion of $\operatorname{Hom}(U, Q)$ in $\operatorname{Hom}\left(\operatorname{Sym}^{2} U, U \otimes Q\right)$, we see that $\mathcal{T}(W, \varphi)$ corresponds to the tangent space of the line.

Note that a nonzero element of $(\mu, \nu) \in \mathcal{T}(U, Q)$ coincides precisely with the first two components of $(\mu, \nu, \lambda) \in \mathcal{V}(U, Q)$ corresponding to $[C(W, \varphi)] \in \mathcal{M}_{[U]}$ via the isomorphism $\theta$ in Proposition 3.10. It follows that the tangent morphism $\tilde{\tau}_{[U]}$ corresponds to the projection $(\mu, \nu, \lambda) \mapsto(\mu, \nu)$. This verifies that the diagram commutes.

\section{ECO polynomials arising from weighted homogeneous polynomials}

In this section, we present some calculations with polynomials and introduce a variety $E^{f}$ associated with a weighted homogeneous polynomial $f$ of a certain type. We will explain the geometric meaning of $E^{f}$ in the next section. 


\section{Jun-Muk Hwang AND Hosung Kim}

Definition 4.1. A homogeneous polynomial of degree $2 m, m \geqslant 1$, in $\mathbb{C}[s, t]$ (in two variables $s$ and $t$ ) is an ECO polynomial if it can be written as the square of a homogeneous polynomial of degree $m$ in $\mathbb{C}[s, t]$. Here ECO stands for Even Contact Order (cf. Definition 5.1).

Recall the following from Proposition 3.3 of [HK13].

Proposition 4.2. For each positive integer $d$, there exist $d$ polynomials in $d$ variables

$$
A_{k}\left(t_{1}, \ldots, t_{d}\right) \in \mathbb{C}\left[t_{1}, \ldots, t_{d}\right], \quad d+1 \leqslant k \leqslant 2 d
$$

with the following properties:

(i) The polynomial $A_{k}\left(t_{1}, \ldots, t_{d}\right)$ is weighted homogeneous of degree $k$ with respect to wt $\left(t_{i}\right)=i$ for each $i=1, \ldots, d$.

(ii) The polynomial in two variables $(s, t)$

$$
s^{2 d}+a_{1} s^{2 d-1} t+\cdots+a_{2 d-1} s t^{2 d-1}+a_{2 d} t^{2 d}
$$

is an ECO polynomial if and only if $a_{k}=A_{k}\left(a_{1}, \ldots, a_{d}\right)$ for each $d+1 \leqslant k \leqslant 2 d$.

(iii) We have

$$
A_{d+i}\left(0, \ldots, 0, a_{d}\right)= \begin{cases}0 & \text { for } i=1, \ldots, d-1, \\ \frac{1}{4} a_{d}^{2} & \text { for } i=d .\end{cases}
$$

Proof. Properties (i) and (ii) are from the statement of Proposition 3.3 of [HK13]. Property (iii) is implicit in the proof therein. It follows essentially from the fact that a polynomial of the form

$$
s^{2 d}+a_{d} s^{d} t^{d}+a_{d+1} s^{d-1} t^{d+1}+\cdots+a_{2 d} t^{2 d}
$$

is an ECO polynomial if and only if

$$
a_{d+1}=\cdots=a_{2 d-1}=0 \quad \text { and } \quad a_{2 d}=\frac{1}{4} a_{d}^{2} .
$$

Let us just check this elementary fact.

Suppose that

$$
s^{2 d}+a_{d} s^{d} t^{d}+a_{d+1} s^{d-1} t^{d+1}+\cdots+a_{2 d} t^{2 d}
$$

is an ECO polynomial. We can find $\left(\sigma_{1}, \ldots, \sigma_{d}\right) \in \mathbb{C}^{d}$ such that

$$
s^{2 d}+a_{d} s^{d} t^{d}+a_{d+1} s^{d-1} t^{d+1}+\cdots+a_{2 d} t^{2 d}=\left(s^{d}+\sigma_{1} s^{d-1} t+\cdots+\sigma_{d} t^{d}\right)^{2} .
$$

For convenience, set $\sigma_{0}=1$. Then for each $k, 1 \leqslant k \leqslant d-1$, we have

$$
\sum_{i=0}^{k} \sigma_{i} \sigma_{k-i}=0 \text {. }
$$

It follows that $\sigma_{1}=\cdots=\sigma_{d-1}=0$. Therefore (4.1) becomes

$$
s^{2 d}+a_{d} s^{d} t^{d}+a_{d+1} s^{d-1} t^{d+1}+\cdots+a_{2 d} t^{2 d}=\left(s^{d}+\sigma_{d} t^{d}\right)^{2},
$$

which implies that

$$
a_{d+1}=\cdots=a_{2 d-1}=0 \quad \text { and } \quad a_{2 d}=\frac{1}{4} a_{d}^{2} .
$$

The proof of the other direction is straightforward. 


\section{Veronese DOUbLe CONES}

Proposition 4.3. Let $f\left(x_{1}, \ldots, x_{n+1}\right)$ be a weighted homogeneous polynomial of degree $2 d$ with respect to $\operatorname{wt}\left(x_{1}\right)=\cdots=\operatorname{wt}\left(x_{n}\right)=1$ and $\operatorname{wt}\left(x_{n+1}\right)=2$. Define $a_{i}^{f}(z)=a_{i}^{f}\left(z_{2}, \ldots, z_{n+2}\right)$ for each $i \in\{0,1, \ldots, 2 d-1,2 d\}$ by

$$
f\left(s, z_{2} t, \ldots, z_{n} t, 2 z_{n+1} s t+z_{n+2} t^{2}\right)=a_{0}^{f}(z) s^{2 d}+a_{1}^{f}(z) s^{2 d-1} t+\cdots+a_{2 d-1}^{f}(z) s t^{2 d-1}+a_{2 d}^{f}(z) t^{2 d} .
$$

Then each $a_{i}^{f}\left(z_{2}, \ldots, z_{n+2}\right)$ is a weighted homogeneous polynomial of degree $i$ with respect to $\operatorname{wt}\left(z_{2}\right)=\cdots \operatorname{wt}\left(z_{n+1}\right)=1$ and $\operatorname{wt}\left(z_{n+2}\right)=2$. In particular, $a_{0}^{f}(z)=f(1,0, \ldots, 0)$ is a constant.

Proof. Putting $s=z_{1} t$, we have

$$
\begin{aligned}
& f\left(z_{1} t, z_{2} t, \ldots, z_{n} t, 2 z_{1} z_{n+1} t^{2}+z_{n+2} t^{2}\right) \\
& \quad=a_{0}^{f}(z) z_{1}^{2 d} t^{2 d}+a_{1}^{f}(z) z_{1}^{2 d-1} t^{2 d}+\cdots+a_{2 d-1}^{f}(z) z_{1} t^{2 d}+a_{2 d}^{f}(z) t^{2 d} .
\end{aligned}
$$

Since $f$ is a weighted homogeneous polynomial of degree $2 d$,

$$
f\left(z_{1} t, z_{2} t, \ldots, z_{n} t, 2 z_{1} z_{n+1} t^{2}+z_{n+2} t^{2}\right)=f\left(z_{1}, \ldots, z_{n}, 2 z_{1} z_{n+1}+z_{n+2}\right) t^{2 d} .
$$

It follows that

$$
f\left(z_{1}, \ldots, z_{n}, 2 z_{1} z_{n+1}+z_{n+2}\right)=a_{0}^{f}(z) z_{1}^{2 d}+a_{1}^{f}(z) z_{1}^{2 d-1}+\cdots+a_{2 d-1}^{f}(z) z_{1}+a_{2 d}^{f}(z) .
$$

Since the left-hand side is a weighted homogeneous polynomial of degree $2 d$ with respect to

$$
\operatorname{wt}\left(z_{1}\right)=\operatorname{wt}\left(z_{2}\right)=\cdots \operatorname{wt}\left(z_{n+1}\right)=1 \text { and } \operatorname{wt}\left(z_{n+2}\right)=2,
$$

each $a_{i}^{f}\left(z_{2}, \ldots, z_{n+2}\right)$ is a weighted homogeneous polynomial of degree $i$ with respect to

$$
\operatorname{wt}\left(z_{2}\right)=\cdots \operatorname{wt}\left(z_{n+1}\right)=1 \text { and } \operatorname{wt}\left(z_{n+2}\right)=2 .
$$

Proposition 4.4. In the setting of Proposition 4.3, assume that the constant $a_{0}^{f}(z)=f(1,0, \ldots, 0)$ is nonzero and denote it by $a_{0}^{f}$. Define for each $i \in\{1, \ldots, d\}$,

$$
B_{d+i}^{f}\left(z_{2}, \ldots, z_{n+2}\right)=B_{d+i}^{f}(z):=\frac{a_{d+i}^{f}(z)}{a_{0}^{f}}-A_{d+i}\left(\frac{a_{1}^{f}(z)}{a_{0}^{f}}, \ldots, \frac{a_{d}^{f}(z)}{a_{0}^{f}}\right),
$$

where $A_{k}$ is as in Proposition 4.2. Then $B_{d+i}^{f}\left(z_{2}, \ldots, z_{n+2}\right)$ is a weighted homogeneous polynomial of degree $d+i$ with respect to $\operatorname{wt}\left(z_{2}\right)=\cdots \operatorname{wt}\left(z_{n+1}\right)=1$ and $\operatorname{wt}\left(z_{n+2}\right)=2$.

Proof. Recall that $A_{k}\left(t_{1}, \ldots, t_{d}\right)$ is a weighted homogeneous polynomial of degree $k$ with respect to $\mathrm{wt}\left(t_{i}\right)=i, 1 \leqslant i \leqslant d$. Thus by Proposition $4.3, A_{k}\left(a_{1}^{f}(z), \ldots, a_{d}^{f}(z)\right)$ is a weighted homogeneous polynomial of degree $k$ with respect to $\operatorname{wt}\left(z_{2}\right)=\cdots \operatorname{wt}\left(z_{n+1}\right)=1$ and $\operatorname{wt}\left(z_{n+2}\right)=2$. This implies the proposition.

Definition 4.5. Fix a basis $\left\{v_{1}, \ldots, v_{n}\right\}$ of an $n$-dimensional vector space $V$ and a nonzero vector $v_{n+1}$ of a one-dimensional vector space $R$. Define $U=\mathbb{C} v_{1}$ and $Q=\mathbb{C} v_{2}+\cdots+\mathbb{C} v_{n}$ such that $V=U \oplus Q$. Let $\left(x_{1}, \ldots, x_{n}, x_{n+1}\right)$ be the linear coordinates on $V \oplus R$ dual to the basis $\left(v_{1}, \ldots, v_{n}, v_{n+1}\right)$. Let $\left(z_{2}, \ldots, z_{n}, z_{n+1}, z_{n+2}\right)$ be the linear coordinates on

$$
\left(\operatorname{Hom}(U, Q) \oplus \operatorname{Hom}\left(\operatorname{Sym}^{2} U, R\right)\right) \oplus \operatorname{Hom}\left(\operatorname{Sym}^{2} U, R\right)
$$

whose values at $(\mu, \nu, \lambda)$ are given by $\mu\left(v_{1}\right)=z_{2}(\mu) v_{2}+\cdots+z_{n}(\mu) v_{n}, \nu\left(v_{1}, v_{1}\right)=z_{n+1}(\nu) v_{n+1}$ and $\lambda\left(v_{1}, v_{1}\right)=z_{n+2}(\lambda) v_{n+1}$.

Let $f\left(x_{1}, \ldots, x_{n+1}\right)$ be as in Proposition 4.4. Let $\mathcal{B}_{d+i}^{f}$ be the weighted hypersurface of degree $d+i$ on the Veronese cone

$$
\mathbb{P}\left(\operatorname{Hom}(U, Q) \oplus \operatorname{Hom}\left(\operatorname{Sym}^{2} U, R\right) ; \operatorname{Hom}\left(\operatorname{Sym}^{2} U, R\right)\right)
$$




\section{Jun-Muk Hwang And Hosung Kim}

defined by the weighted homogeneous polynomial $B_{d+i}^{f}\left(z_{2}, \ldots, z_{n+2}\right)$ of degree $d+i$ with respect to $\operatorname{wt}\left(z_{2}\right)=\cdots=\operatorname{wt}\left(z_{n+1}\right)=1$ and $\operatorname{wt}\left(z_{n+2}\right)=2$. We define the subvariety

$$
E^{f} \subset \mathbb{P}\left(\operatorname{Hom}(U, Q) \oplus \operatorname{Hom}\left(\operatorname{Sym}^{2} U, R\right) ; \operatorname{Hom}\left(\operatorname{Sym}^{2} U, R\right)\right)
$$

as the set-theoretic intersection of the hypersurfaces $\left\{\mathcal{B}_{d+i}^{f} \mid i=1, \ldots, d\right\}$.

The following lemma is a direct translation of Proposition 4.2 into the terminology of Definition 4.5.

Lemma 4.6. Let $f\left(x_{1}, \ldots, x_{n+1}\right)$ be a weighted homogeneous polynomial of degree $2 d$ with respect to $\operatorname{wt}\left(x_{1}\right)=\cdots=\operatorname{wt}\left(x_{n}\right)=1$ and $\operatorname{wt}\left(x_{n+1}\right)=2$ satisfying $f(1,0, \ldots, 0) \neq 0$. Then the homogeneous polynomial in $\mathbb{C}[s, t]$

$$
f\left(s, z_{2} t, \ldots, z_{n} t, 2 z_{n+1} s t+z_{n+2} t^{2}\right)
$$

is an ECO polynomial if and only if the element

$$
\left(z_{2}, \ldots, z_{n}, z_{n+1}, z_{n+2}\right) \in\left(\operatorname{Hom}(U, Q) \oplus \operatorname{Hom}\left(\operatorname{Sym}^{2} U, R\right)\right) \oplus \operatorname{Hom}\left(\operatorname{Sym}^{2} U, R\right)
$$

in the coordinates of Definition 4.5 belongs to a point of $E^{f}$.

Proposition 4.7. A general weighted homogeneous polynomial $f\left(x_{1}, \ldots, x_{n+1}\right)$ of degree $2 d$ with respect to $\operatorname{wt}\left(x_{1}\right)=\cdots=\operatorname{wt}\left(x_{n}\right)=1$ and $\operatorname{wt}\left(x_{n+1}\right)=2$ satisfying $f(1,0, \ldots, 0) \neq 0$ has the following properties:

(i) The locus $f=0$ defines a smooth weighted hypersurface of degree $2 d$ in $\mathbb{P}(V ; R)$.

(ii) The subvariety $E^{f}$ in Definition 4.5 is disjoint from the locus of $\left(z_{2}=\cdots=z_{n}=0\right)$ in the Veronese cone

$$
\mathbb{P}\left(\operatorname{Hom}(U, Q) \oplus \operatorname{Hom}\left(\operatorname{Sym}^{2} U, R\right) ; \operatorname{Hom}\left(\operatorname{Sym}^{2} U, R\right)\right) .
$$

In other words, it belongs to $\mathcal{V}(U, Q)$ (see Notation 3.8).

(iii) The subvariety $E^{f}$ is a smooth weighted complete intersection of multi-degree $(d+1$, $d+2, \ldots, 2 d)$ in $\mathcal{V}(U, Q)$. In particular, it is nonempty if and only if $d \leqslant n$.

Proof. Property (i) is obvious. Since properties (ii) and (iii) are open conditions, we may verify them for a special $f\left(x_{1}, \ldots, x_{n+1}\right)$. Let us consider polynomials of the following type:

$$
\begin{aligned}
& f\left(x_{1}, \ldots, x_{n+1}\right) \\
& \quad=x_{1}^{2 d}+x_{1}^{d-1} h_{d+1}\left(x_{2}, \ldots, x_{n}\right)+\cdots+x_{1} h_{2 d-1}\left(x_{2}, \ldots, x_{n}\right)+h_{2 d}\left(x_{2}, \ldots, x_{n}\right)+x_{n+1}^{d}
\end{aligned}
$$

where $h_{i}\left(x_{2}, \ldots, x_{n}\right)$ is a homogeneous polynomial of degree $i$ in $x_{2}, \ldots, x_{n}$. From

$$
\begin{aligned}
& f\left(1, z_{2} t, \ldots, z_{n} t, 2 z_{n+1} t+z_{n+2} t^{2}\right) \\
& \quad=1+t^{d+1} h_{d+1}\left(z_{2}, \ldots, z_{n}\right)+\cdots+t^{2 d} h_{2 d}\left(z_{2}, \ldots, z_{n}\right)+t^{d} \sum_{i=0}^{d} 2^{d-i}\left(\begin{array}{c}
d \\
i
\end{array}\right) z_{n+1}^{d-i} z_{n+2}^{i} t^{i} \\
& \quad=1+2^{d} z_{n+1}^{d} t^{d}+\sum_{i=1}^{d}\left\{h_{d+i}\left(z_{2}, \ldots, z_{n}\right)+2^{d-i}\left(\begin{array}{c}
d \\
i
\end{array}\right) z_{n+1}^{d-i} z_{n+2}^{i}\right\} t^{d+i} \\
& =a_{0}^{f}(z)+a_{1}^{f}(z) t+\cdots+a_{2 d}^{f}(z) t^{2 d},
\end{aligned}
$$

we have

$$
a_{0}^{f}(z)=1, \quad a_{1}^{f}(z)=\cdots=a_{d-1}^{f}(z)=0, \quad a_{d}^{f}(z)=2^{d} z_{n+1}^{d}
$$


and

$$
a_{d+i}^{f}(z)=h_{d+i}\left(z_{2}, \ldots, z_{n}\right)+2^{d-i}\left(\begin{array}{c}
d \\
i
\end{array}\right) z_{n+1}^{d-i} z_{n+2}^{i} \quad \text { for } i=1, \ldots, d .
$$

Using Proposition 4.2 (iii), we have

$$
B_{d+i}^{f}(z)= \begin{cases}h_{d+i}\left(z_{2}, \ldots, z_{n}\right)+2^{d-i}\left(\begin{array}{c}
d \\
i
\end{array}\right) z_{n+1}^{d-i} z_{n+2}^{i} & \text { for } i=1, \ldots, d-1, \\
h_{2 d}\left(z_{2}, \ldots, z_{n}\right)-4^{d-1} z_{n+1}^{2 d}+z_{n+2}^{d} & \text { for } i=d .\end{cases}
$$

From these equations for $\mathcal{B}_{d+i}^{f}$, we see that property (ii) is true for any choice of $h_{d+i}, 1 \leqslant i \leqslant d$.

Now we choose $h_{2 d}\left(z_{2}, \ldots, z_{n}\right)=z_{2}^{2 d}+\cdots+z_{n}^{2 d}$. Then the subscheme $Z$ defined by the equation

$$
B_{2 d}^{f}\left(z_{2}, \ldots, z_{n+2}\right)=z_{2}^{2 d}+\ldots+z_{n}^{2 d}-4^{d-1} z_{n+1}^{2 d}+z_{n+2}^{d}=0
$$

is a smooth weighted hypersurface of degree $2 d$ in the Veronese cone

$$
\mathbb{P}\left(\operatorname{Hom}(U, Q) \oplus \operatorname{Hom}\left(\operatorname{Sym}^{2} U, R\right) ; \operatorname{Hom}\left(\operatorname{Sym}^{2} U, R\right)\right)
$$

disjoint from the vertex of the Veronese cone. Note that this subscheme $Z$ is independent of the choice of $h_{d+i}, 1 \leqslant i \leqslant d-1$. We will show that property (iii) is true under a general choice of $h_{d+1}, \ldots, h_{2 d-1}$.

For simplicity, let us denote by

$$
\psi_{Z}: Z \rightarrow \mathbb{P}^{n-1}=\mathbb{P}\left(\operatorname{Hom}(U, Q) \oplus \operatorname{Hom}\left(\operatorname{Sym}^{2} U, R\right)\right)
$$

the restriction to $Z$ of the morphism

$$
\Psi_{\operatorname{Hom}(U, Q) \oplus \operatorname{Hom}\left(\operatorname{Sym}^{2} U, R\right)}^{\operatorname{Hom}\left(\mathrm{Sym}^{2} U, R\right)}
$$

in Notation 3.8. Note that for each $i=1, \ldots, d-1$,

$$
\left.h_{d+i}\right|_{Z} \in \psi_{Z}^{*} H^{0}\left(\mathbb{P}^{n-1}, \mathcal{O}_{\mathbb{P}^{n-1}}(d+i)\right),
$$

while

$$
B_{d+i}^{f}\left|Z=\left(h_{d+i}\left(z_{2}, \ldots, z_{n}\right)+2^{d-i}\left(\begin{array}{c}
d \\
i
\end{array}\right) z_{n+1}^{d-i} z_{n+2}^{i}\right)\right|_{Z} \in H^{0}\left(Z, \psi_{Z}^{*} \mathcal{O}_{\mathbb{P}^{n-1}}(d+i)\right) .
$$

By the Bertini theorem, if $h_{d+1}, \ldots, h_{2 d-1}$ are general, the scheme-theoretic intersection

$$
Z \cap \bigcap_{i=1}^{d-1}\left(h_{d+i}=0\right)
$$

is smooth, of codimension $d-1$ in $Z$ and contained in $\mathcal{V}(U, Q)$. Thus the scheme-theoretic intersection

$$
Z \cap \bigcap_{i=1}^{d-1}\left(h_{d+i}\left(z_{2}, \ldots, z_{n}\right)+2^{d-i}\left(\begin{array}{l}
d \\
i
\end{array}\right) t z_{n+1}^{d-i} z_{n+2}^{i}=0\right)
$$

is smooth, of codimension $d-1$ in $Z$ and contained in $\mathcal{V}(U, Q)$ for a general $t \in \mathbb{C}$. It follows that if $h_{d+1}, \ldots, h_{2 d-1}$ are general, then

$$
E^{f}=Z \cap \bigcap_{i=1}^{d-1}\left(h_{d+i}\left(z_{2}, \ldots, z_{n}\right)+2^{d-i}\left(\begin{array}{l}
d \\
i
\end{array}\right) z_{n+1}^{d-i} z_{n+2}^{i}=0\right)
$$

is a smooth weighted complete intersection of multi-degree $(d+1, d+2, \ldots, 2 d)$ contained in $\mathcal{V}(U, Q)$. This has property (iii). 


\section{Jun-Muk Hwang And Hosung Kim}

\section{ECO conics on a Veronese cone}

Definition 5.1. Let $Z \subset \mathbb{P}(V ; R)$ be a weighted hypersurface of degree $2 d$ for $d \geqslant 1$. We say that a conic $C$ in $\mathbb{P}(V ; R)$ is an ECO (Even Contact Order) conic with respect to $Z$ if $C \not \subset Z$ and the local intersection number at each point of $C \cap Z$ is even. For a point $y \in \mathbb{P}(V ; R)$, denote by $\mathcal{E}_{y}^{Z} \subset \mathcal{M}_{y}$ the subvariety consisting of ECO conics through $y$. Here $\mathcal{M}_{y}$ is as in Proposition 3.6.

Proposition 5.2. Using the linear coordinates of Definition 4.5, let $Z \subset \mathbb{P}(V ; R)$ be the weighted hypersurface of degree $2 d$ defined by a weighted homogeneous polynomial $f$ of degree $2 d$ with respect to $\operatorname{wt}\left(x_{1}\right)=\cdots=\operatorname{wt}\left(x_{n}\right)=1$ and $\operatorname{wt}\left(x_{n+1}\right)=2$. Using the decomposition $V=U \oplus Q$, we have the isomorphism $\theta: \mathcal{V}(U, Q) \cong \mathcal{M}_{[U]}$ by Proposition 3.10. Suppose that $f$ is general in the sense of Proposition 4.7. Then the variety $\mathcal{E}_{[U]}^{Z} \subset \mathcal{M}_{[U]}$ of Definition 5.1 is exactly the $\theta$-image of the variety $E^{f} \subset \mathcal{V}(U, Q)$ of Proposition 4.7.

Proof. Recall from Proposition 3.10 that $\theta$ sends a point $[(\mu, \nu, \lambda)] \in \mathcal{V}(U, Q)$ to a conic $C(W, \varphi)$, where

$$
W=\left\{s v_{1}+t \mu\left(v_{1}\right)=s v_{1}+t z_{2}(\mu) v_{2}+\ldots+t z_{n}(\mu) v_{n} \mid(s, t) \in \mathbb{C}^{2}\right\}
$$

and for any $(s, t) \in \mathbb{C}^{2}$,

$$
\begin{aligned}
\varphi\left(s v_{1}+t \mu\left(v_{1}\right), s v_{1}+t \mu\left(v_{1}\right)\right) & =2 s t \varphi\left(v_{1}, \mu\left(v_{1}\right)\right)+t^{2} \varphi\left(\mu\left(v_{1}\right), \mu\left(v_{1}\right)\right) \\
& =2 s t \nu\left(v_{1}, v_{1}\right)+t^{2} \lambda\left(v_{1}, v_{1}\right) \\
& =\left(2 s t z_{n+1}(\nu)+t^{2} z_{n+2}(\lambda)\right) v_{n+1} .
\end{aligned}
$$

Consequently, $C(W, \varphi)=\{[(w, \varphi(w, w))] \in \mathbb{P}(V ; R) \mid w \in W \backslash\{0\}\}$ is equal to

$$
\left\{\left[\left(s v_{1}+t z_{2}(\mu) v_{2}+\cdots+t z_{n}(\mu) v_{n},\left(2 s t z_{n+1}(\nu)+t^{2} z_{n+2}(\lambda)\right) v_{n+1}\right)\right] \mid(s, t) \in \mathbb{C}^{2} \backslash\{(0,0)\}\right\} .
$$

Therefore $C(W, \varphi)$ is an ECO conic with respect to $Z$ if and only if

$$
f\left(s, z_{2}(\mu) t, \ldots, z_{n}(\mu) t, 2 z_{n+1}(\nu) s t+z_{n+2}(\lambda) t^{2}\right)
$$

is an ECO polynomial in $(s, t)$. By Lemma 4.6, this is the case if and only if the point

$$
\left(z_{2}(\mu), \ldots, z_{n}(\mu), z_{n+1}(\nu), z_{n+2}(\lambda)\right) \in \operatorname{Hom}(U, Q) \oplus \operatorname{Hom}\left(\operatorname{Sym}^{2} U, R\right) \oplus \operatorname{Hom}\left(\operatorname{Sym}^{2} U, R\right)
$$

belongs to $E^{f} \subset \mathcal{V}(U, Q)$. This completes the proof.

Proposition 5.3. Let $Z \subset \mathbb{P}(V ; R)$ be as in Proposition 5.2, defined by a general weighted homogeneous polynomial $f$ of degree $2 d$ in the sense of Proposition 4.7. Assume $2 \leqslant d \leqslant n / 2$. Let $y \in \mathbb{P}(V ; R)$ be a general point. Then the tangent morphism

$$
\tau_{y}^{Z}: \mathcal{E}_{y}^{Z} \rightarrow \mathbb{P} T_{y}(\mathbb{P}(V ; R))
$$

defined by the restriction of $\tilde{\tau}_{y}$ in Notation 3.9 is not an immersion.

Proof. Fix a general $f$ in the sense of Proposition 4.7. Let $Z \subset \mathbb{P}(V ; R)$ be the hypersurface defined by $f$. Via the isomorphism $\theta: E^{f} \cong \mathcal{E}_{[U]}^{Z}$ of Proposition 5.2, the morphism $\tau_{[U]}^{Z}$ is the restriction of the morphism $\Psi(U, Q)$ of Proposition 3.11 to $E^{f}$. By Proposition 4.7, the variety $E^{f}$ is a smooth weighted complete intersection of multi-degree $(d+1, d+2, \ldots, 2 d)$. By applying Proposition 2.9 to the $n$-dimensional Veronese cone

$$
\mathbb{P}\left(\operatorname{Hom}(U, Q) \oplus \operatorname{Hom}\left(\operatorname{Sym}^{2} U, R\right) ; \operatorname{Hom}\left(\operatorname{Sym}^{2} U, R\right)\right),
$$

we see that $\tau_{[U]}^{Z}$ is not an immersion by our assumption $2 \leqslant d \leqslant n / 2$. 


\section{VERONESE DOUBLE CONES}

Now consider the $G$-action on $\mathbb{P}(V ; R)$ in Proposition 2.6. For an element $g \in G$, the hypersurface $g \cdot Z$ is also a weighted hypersurface of degree $2 d$ by Proposition 2.6 (iii). We have just proved that $\tau_{[U]}^{Z}$ is not an immersion for a general $Z$. Thus there exists a Zariski open subset $G^{*} \subset G$ containing the identity element such that the morphism $\tau_{[U]}^{g \cdot Z}$ is not an immersion for any $g \in G^{*}$. By the $g$-action, the morphism $\tau_{[U]}^{g \cdot Z}$ is isomorphic to the morphism $\tau_{g^{-1} \cdot[U]}^{Z}$. Thus $\tau_{g^{-1} \cdot[U]}^{Z}$ is not an immersion. By Proposition 2.6 (ii), the set $\left\{g^{-1} \cdot[U], g \in G^{*}\right\}$ covers a Zariski open subset of $\mathbb{P}(V ; R)^{o}$. It follows that $\tau_{y}^{Z}$ is not an immersion for a general point $y \in \mathbb{P}(V ; R)$.

\section{Minimal rational curves on a Veronese double cone}

Definition 6.1. Let $d \geqslant 3$ be an odd integer. Let $V, R$ and $T$ be $\mathbb{C}$-vector spaces of dimensions $\operatorname{dim} V=n \geqslant 3$ and $\operatorname{dim} R=\operatorname{dim} T=1$. Consider the $\mathbb{C}^{\times}$-action on $V \oplus R \oplus T$ where $c \in \mathbb{C}^{\times}$ acts by $(v, r, t) \mapsto\left(c v, c^{2} r, c^{d} t\right)$. The quotient space of $(V \oplus R \oplus T) \backslash(0 \oplus 0 \oplus 0)$ by this $\mathbb{C}^{\times}$-action is a projective variety, denoted by $\mathbb{P}(V ; R ; T)$.

DeFinition 6.2. Let $x_{1}, \ldots, x_{n}$ be linear coordinates on $V$ and let $x_{n+1}$ (respectively, $x_{n+2}$ ) be a nonzero linear functional on $R$ (respectively, $T$ ). We will use $\left(x_{1}, \ldots, x_{n}, x_{n+1}, x_{n+2}\right)$ as a linear coordinate system on $V \oplus R \oplus T$. Let $f\left(x_{1}, \ldots, x_{n}, x_{n+1}\right)$ be a weighted homogeneous polynomial of degree $2 d$ with respect to $\operatorname{wt}\left(x_{1}\right)=\cdots=\operatorname{wt}\left(x_{n}\right)=1$ and $\operatorname{wt}\left(x_{n+1}\right)=2$. Consider the hypersurface in $V \oplus R \oplus T$

$$
\widehat{X^{f}}:=\left\{\left(x_{1}, \ldots, x_{n}, x_{n+1}, x_{n+2}\right) \in V \oplus R \oplus T, f\left(x_{1}, \ldots, x_{n}, x_{n+1}\right)=x_{n+2}^{2}\right\} .
$$

The hypersurface $X^{f} \subset \mathbb{P}(V ; R ; T)$ consisting of $\mathbb{C}^{\times}$-equivalence classes of points on $\widehat{X^{f}}$ is called the Veronese double cone associated with $f$.

The next three propositions are well known. We recall the proofs for readers' convenience.

Proposition 6.3. In Definition 6.2, assume that the hypersurface $Z \subset \mathbb{P}(V ; R)$ defined by $f=0$ is nonsingular and disjoint from $\mathbf{v t x}(V ; R)$. Then the following hold:

(i) The projective variety $X^{f}$ is nonsingular.

(ii) The rational map $\widehat{\chi}: \mathbb{P}(V ; R ; T)-\rightarrow \mathbb{P}(V ; R)$ defined by the projection $(V \oplus R \oplus T) \rightarrow(V \oplus R)$ induces a morphism $\chi: X^{f} \rightarrow \mathbb{P}(V ; R)$, which is a finite morphism of degree two and is unramified outside $Z \cup \operatorname{vtx}(V ; R) \subset \mathbb{P}(V ; R)$.

Proof. The hypersurface

$$
\widehat{X^{f}} \backslash(0 \oplus 0 \oplus 0) \subset V \oplus R \oplus T
$$

is nonsingular by the assumption that $Z$ is nonsingular. The variety $X^{f}$ is the quotient of $\widehat{X^{f}} \backslash(0 \oplus 0 \oplus 0)$ by the $\mathbb{C}^{\times}$-action given by

$$
c \in \mathbb{C}^{\times} \quad \text { acting by } \quad(v, r, t) \mapsto\left(c v, c^{2} r, c^{d} t\right) .
$$

To prove that $X_{f}$ is nonsingular, it suffices to show that this $\mathbb{C}^{\times}$-action on $\widehat{X^{f}} \backslash(0 \oplus 0 \oplus 0)$ is free, that is, it has trivial stabilizers at all points of $\widehat{X^{f}} \backslash(0 \oplus 0 \oplus 0)$. Because $d$ is odd, the only points of $V \oplus R \oplus T$ with nontrivial stabilizers are the points on $0 \oplus R \oplus 0$ and $0 \oplus 0 \oplus T$. But $\widehat{X^{f}} \backslash(0 \oplus 0 \oplus 0)$ is disjoint from these points. We conclude that the quotient $X^{f}$ is nonsingular, proving result (i). 


\section{Jun-Muk Hwang AND Hosung Kim}

The indeterminacy locus of the rational map $\widehat{\chi}$ is the subvariety $\mathbb{P} T \subset \mathbb{P}(V ; R ; T)$ corresponding to $0 \oplus 0 \oplus T$. Since $X^{f} \subset \mathbb{P}(V ; R ; T)$ is disjoint from $\mathbb{P} T$, the rational map $\widehat{\chi}$ induces a morphism $\chi: X^{f} \rightarrow \mathbb{P}(V ; R)$. For a point $(v, r)=\left(x_{1}, \ldots, x_{n}, x_{n+1}\right) \in V \oplus R$ with $f\left(x_{1}, \ldots, x_{n}, x_{n+1}\right) \neq 0$, its inverse image $\widehat{\chi}^{-1}((v, r))$ consists of two distinct points

$$
\left(x_{1}, \ldots, x_{n}, x_{n+1}, x_{n+2}\right) \text { and }\left(x_{1}, \ldots, x_{n}, x_{n+1},-x_{n+2}\right) \text {, }
$$

where $x_{n+2} \in \mathbb{C}$ is a solution of $x_{n+2}^{2}=f\left(x_{1}, \ldots, x_{n}, x_{n+1}\right)$. These two distinct points of $\widehat{X^{f}}$ are sent to the same point of $X^{f}$ if and only if there is a $c \in \mathbb{C}^{\times}$with

$$
x_{1}=c x_{1}, \ldots, x_{n}=c x_{n}, \quad x_{n+1}=c^{2} x_{n+1} \quad \text { and } \quad x_{n+2}=-c^{d} x_{n+2} .
$$

The only possibility is $x_{1}=\cdots=x_{n}=0$ and $c=-1$. Then $(v, r)=\mathbf{v t x}(V ; R)$. Thus $\chi$ is 2-to- 1 outside $Z$ and $\operatorname{vtx}(V ; R)$. This completes the proof of result (ii).

Proposition 6.4. For a homogeneous polynomial $g\left(x_{1}, \ldots, x_{n}\right)$ of degree one in the coordinate system in Definition 6.2, the equation $g\left(x_{1}, \ldots, x_{n}\right)=0$ defines a Weil divisor $D \subset \mathbb{P}(V ; R ; T)$. Let $X^{f} \subset \mathbb{P}(V ; R ; T)$ be as in Proposition 6.3 , so that $X^{f} \cap D$ determines a line bundle $L$ on $X^{f}$. Then $\operatorname{Pic}\left(X_{f}\right) \cong \mathbb{Z} L$ and $K_{X^{f}}=(d-n-2) L$.

Proof. This follows from Theorems 3.2.4. and 3.3.4 of [Dol82], where the line bundle $L$ is denoted by $\mathcal{O}(1)$.

Proposition 6.5. Let $\chi: X^{f} \rightarrow \mathbb{P}(V ; R)$ be the morphism in Proposition 6.3 and let $\iota: \mathbb{P}(V ; R) \rightarrow \mathbb{P}\left(\operatorname{Sym}^{2} V \oplus R\right)$ be the morphism in Proposition 2.2. Then $L^{\otimes 2}=(\iota \circ \chi)^{*} \xi$, where $\xi$ is the hyperplane bundle on $\mathbb{P}\left(\mathrm{Sym}^{2} V \oplus R\right)$.

Proof. In the notation of Proposition 6.4, $g^{2}$ corresponds to a section of $\xi$. Thus $L^{\otimes 2}=$ $(\iota \circ \chi)^{*} \xi$.

DeFinition 6.6. In the setting of Proposition 6.3, a rational curve $\ell \subset X^{f}$ is called a minimal rational curve on $X^{f}$ if $L \cdot \ell=1$, where $L$ is the line bundle in Proposition 6.4. For a general point $x \in X^{f}$, we denote by $\mathcal{K}_{x}$ the normalization of the subscheme of $\operatorname{RatCurves}^{n}\left(X^{f}\right)$ parametrizing all minimal rational curves on $X^{f}$ passing through $x$. It is known that if $\mathcal{K}_{x}$ is nonempty, it is a disjoint union of finitely many nonsingular projective varieties of dimension $n-d$ (see II.3.11.5 of $[\mathrm{Kol} 96])$.

Proposition 6.7. Assume $n \geqslant d$. In Proposition 6.3, let $x$ be a general point on $X^{f}$ such that $y:=\chi(x) \in \mathbb{P}(V ; R) \backslash Z$. Let $C \subset \mathbb{P}(V ; R)$ be an ECO conic passing through $y$ with respect to $Z$ in the sense of Definition 5.1. Then $\chi^{-1}(C)$ consists of two smooth minimal rational curves meeting each other at the points over $C \cap Z$. In particular, there exists a unique minimal rational curve $\ell \subset X^{f}$ passing through $x$ satisfying $\chi(\ell)=C$. Conversely, if $\ell \subset X^{f}$ is a minimal rational curve through $x$, then $\chi(\ell)$ is an ECO conic on $\mathbb{P}(V ; R)$ through $y$. Consequently, there exists a natural isomorphism $\chi_{*}: \mathcal{K}_{x} \cong \mathcal{E}_{y}^{Z}$ of nonsingular varieties.

Proof. The proof is a slight variation of that of Proposition 2.4 in [HK13].

Let $\ell \subset X^{f}$ be an irreducible curve through $x$ such that $C:=\chi(\ell) \subset \mathbb{P}(V ; R)$ is an ECO conic with respect to $Z$. Suppose that $\left.\chi\right|_{\ell}: \ell \rightarrow C$ is not birational; that is, $\ell=\chi^{-1}(C)$. Let $\tilde{\ell}$ be the normalization of $\ell$. For a point $z \in C \cap Z$, let $t$ be a local uniformizing parameter on $C$ at $z$ and let $r_{z}$ be the local intersection number of $C$ and $Z$ at $z$. Then the germ of $\ell$ at $\chi^{-1}(z)$ is analytically defined by the equation $s^{2}=t^{r_{z}}$. Since $r_{z}$ is even for any choice of $z \in C \cap Z$, the composition of the normalization morphism $\tilde{\ell} \rightarrow \ell$ and $\left.\chi\right|_{\ell}: \ell \rightarrow C$ induces a morphism $\tilde{\ell} \rightarrow C$ 


\section{VERONESE DOUBLE CONES}

of degree two without ramification point, giving a contradiction. Thus $\left.\chi\right|_{\ell}: \ell \rightarrow C$ is birational and $\ell \cdot L=1$ by Proposition 6.5. It follows that $\ell$ is a minimal rational curve.

Conversely, let $\ell \subset X^{f}$ be a minimal rational curve through $x$. By Proposition 6.5, the cycle $\chi_{*}(\ell)$ is either a conic or a double line on $\mathbb{P}(V ; R)$. By Proposition 3.2 , a line $m$ through a general point $y \in \mathbb{P}(V ; R)$ intersects the smooth hypersurface $Z \subset \mathbb{P}(V ; R)$ transversally at $d \geqslant 3$ distinct points. Thus $\chi^{-1}(m)$ is irreducible and cannot be a rational curve. It follows that $\chi_{*}(\ell)$ is a conic $C \subset \mathbb{P}(V ; R)$ and $\chi^{-1}(C)$ has two irreducible components. We claim that $C$ is an ECO conic with respect to $Z$. For each point $z \in C \cap Z$, if the local intersection number $r_{z}$ of $C$ and $Z$ at $z$ is odd, the germ of $\chi^{-1}(C)$ over $z$ is irreducible (for example, by the equation $s^{2}=t^{r_{z}}$ in the previous paragraph). This is impossible because $\chi^{-1}(C)$ has two distinct components. Thus $r_{z}$ is even at every $z \in C \cap Z$ and $C$ is an ECO conic with respect to $Z$.

We are ready to prove Theorem 1.3.

Proof of Theorem 1.3. Propositions 5.2 and 6.7 imply that $\mathcal{K}_{x}$ is isomorphic to $E^{f}$, which is a smooth weighted complete intersection of multi-degree $(d+1, d+2, \ldots, 2 d)$ in $\mathcal{V}(U, Q)$ by Proposition 4.7. Therefore $\mathcal{K}_{x}$ is nonempty when $n \geqslant d$, and irreducible when $n>d$, because it is smooth and connected. Thus minimal rational curves form the union of finitely many minimal dominating families of rational curves on $X^{f}$ if $n \geqslant d$, and they form a single minimal dominating family if $n>d$. We have a commuting diagram

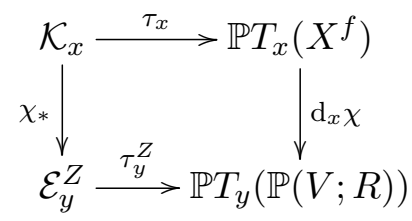

The first vertical arrow is an isomorphism by Proposition 6.7. As $\chi$ is unramified at $x$, the second vertical arrow $\mathrm{d}_{x} \chi$ is an isomorphism. If $n \geqslant 2 d$, the morphism $\tau_{y}^{Z}$ is not an immersion by Proposition 5.3. Thus the morphism $\tau_{x}$ is not an immersion.

\section{REFERENCES}

CD12 C. Casagrande and S. Druel, Locally unsplit families of rational curves of large anticanonical degree on Fano manifolds, arXiv:1212.5083.

Dol82 I. Dolgachev, Weighted projective varieties, in Group actions and vector fields (Vancouver, B.C., 1981), Lecture Notes in Math., vol. 956, Springer, Berlin, 1982, 34-71.

Hwa01 J.-M. Hwang, Geometry of minimal rational curves on Fano manifolds, in School on vanishing Theorems and Effective Results in Algebraic Geometry (Trieste, 2000), ICTP Lect. Notes, vol. 6, Abdus Salam Int. Cent. Theoret. Phys., Trieste, 2001, 335-393.

Hwa12 J.-M. Hwang, Geometry of varieties of minimal rational tangents, in Current Developments in Algebraic Geometry, MSRI Publications, vol. 59, Cambridge University Press, New York, 2012, $197-222$.

HK13 J.-M. Hwang and H. Kim, Varieties of minimal rational tangents on double covers of projective space, Math. Zeit. 275 (2013) 109-125. http://dx.doi.org/10.1007/s00209-012-1125-6

HM04 J.-M. Hwang and N. Mok, Birationality of the tangent map for minimal rational curves, Asian J. Math. 8 (2004), 51-64. http://dx.doi.org/10.4310/AJM.2004.v8.n1.a6

Keb02 S. Kebekus, Families of singular rational curves, J. Algebraic Geom. 11 (2002), 245-256. http: //dx.doi.org/10.1090/S1056-3911-01-00308-3 


\section{Jun-Muk Hwang And Hosung Kim}

Kol96 J. Kollár, Rational curves on algebraic varieties, Ergebnisse der Mathematik und ihrer Grenzgebiete, vol. 32, Springer, 1996.

Jun-Muk Hwang jmhwang@kias.re.kr

Korea Institute for Advanced Study, Hoegiro 87, Seoul, 130-722, Korea

Hosung Kim hosung@kias.re.kr

Korea Institute for Advanced Study, Hoegiro 87, Seoul, 130-722, Korea 